\title{
O acesso à medicamentos: aspectos da judicialização da saúde
}

\section{The access to medicines: aspects of the judicialization of health}

\author{
Maria da Graça Lepre Hawerroth ${ }^{1}$. Lucas Yuji Sonoda ${ }^{1}$. Raquel Dully Andrade ${ }^{1}$. Jaqueline Silva Santos ${ }^{2}$. \\ Maria Ambrosina Cardoso Maia ${ }^{1}$. \\ 1 Universidade do Estado de Minas Gerais (UEMG), Passos, Minas Gerais, Brasil. 2 Secretaria de Estado de Saúde, Superintendência \\ Regional de Saúde, Passos, Minas Gerais, Brasil.
}

\section{RESUMO}

Introdução: a judicialização da saúde é um fenômeno mundial, ético-filosófico e social em crescimento, que impacta de forma negativa nas finanças públicas e demonstra as mazelas do sistema de saúde. Objetivo: realizar uma revisão da literatura acerca da judicialização da saúde em que os insumos requisitados são medicamentos. Revisão de literatura: foi realizada uma revisão narrativa da literatura nas bases de dados Pubmed, Medline e Lilacs, abordando artigos publicados nos últimos 10 anos que abordassem a judicialização do acesso a medicamentos. Retornaram a busca 354 resultados e após os critérios de inclusão e exclusão, foram analisadas 27 publicações. Discussão: considerando a utilização da via judicial para acesso a medicamentos, os estudos apontam diversos desdobramentos, como o impacto financeiro, aspectos éticos e sociais, utilização de serviços públicos e privados, presença de medicamentos em listas oficiais de distribuição, além do aumento explosivo do número de casos relatados nos últimos anos. Conclusão: a judicialização da saúde apresenta discussões paradoxais quanto ao seu impacto negativo e a necessidade de atualização das listas oficiais de medicamentos.

Palavras-chave: Direito à saúde. Judicialização da saúde. Acesso a medicamentos.

\begin{abstract}
Introduction: The judicialization of health is a rising social, ethical and philophical worldwide phenomenon that has a negative impact on public budgets and shows the dysfunction of the health system. Objective: Accomplish a literature review about the judicialization of health, which the required elements are drugs. Literature review: A review of the literature was performed using 3 databases: Pubmed, Medline and Lilacs, approaching papers published in the last 10 years that are related to the judicialization of health on access to medicines. The search found 354 articles e after the inclusion and exclusion criteria, 27 papers were analyzed. Discussion: Considering the use of the judicial pathway on access to medicines, the results point out to many topics such as the financial impact, social and ethical aspects, application of public and private services, availability of essential medicines in pharmaceutical assistance programme, and the explosive rise of cases in the last years. Conclusion: Therefore, it can be concluded that such phenomenon presents paradoxical discussions about its negative financial impacts and the need for pharmaceutical assistance program update.
\end{abstract}

Keywords: Right to health. Health's judicialization. Access to Medications.

Autor correspondente: Maria da Graça Lepre Hawerroth, Rua dos Brandões, 383, Passos, Minas Gerais, Brasil. CEP: 37900-104. Telefone: +55 44 99827-1776. E-mail: maria.2136997@discente.uemg.br

Conflito de interesses: Não há qualquer conflito de interesses por parte de qualquer um dos autores.

Recebido em: 06 Set 2020; Revisado em: 01 Mar 2021; Aceito em: 02 Mar 2021. 


\section{INTRODUÇÃO}

No Brasil, o artigo 196 da Constituição da República de 1988 afirma que a saúde é um direito de toda pessoa, sendo dever do Estado a formulação de políticas públicas para garanti-la. ${ }^{1}$ Entretanto, muitas vezes os cidadãos recorrem à judicialização como forma de garantir seus direitos inalienáveis, como o acesso a serviços e medicamentos. Nesse cenário, a judicialização da saúde aborda questões ético-filosóficas configurando como um fenômeno que envolve as esferas jurídicas, médicas, sanitárias e comerciais., ${ }^{2,3}$

A judicialização para o acesso à medicamentos é uma realidade observada e estudada no Brasil. ${ }^{4}$ Em parte, essa busca ao Poder Judiciário pode ser decorrente das dificuldades de acesso aos medicamentos pelos usuários do Sistema Único de Saúde (SUS), ou seja, um sistema de saúde disfuncional que falha em atender suas demandas. ${ }^{5}$

Aqui, apreende-se que o fenômeno da judicialização da saúde é complexo e multidisciplinar, apresenta aspectos positivos e negativos, por ser considerado uma consolidação dos direitos inalienáveis dos cidadãos e, ao mesmo tempo, ter a possibilidade de trazer impactos ao orçamento público de forma desfavorável. ${ }^{5,6}$

Além disso, é preciso reconhecer que o processo de implementação do SUS têm se deparado com constantes desafios, problemas e fragilidades ${ }^{7}$ e emerge a necessidade de renovação e atualização de recursos tecnológicos do âmbito da saúde, considerando-se as disparidades entre oferta de recursos e o volume necessário. ${ }^{2}$ Destarte, o subfinanciamento do $\mathrm{SUS}^{7}$ é uma realidade que pode ser agravada pela crise vivenciada pelo Brasil, com possíveis reflexos na judicialização da saúde.

O entendimento é que o fenômeno da judicialização da saúde funciona como garantidor de direitos violados, todavia, há de se reconhecer que pode resultar em impactos financeiros para o SUS. Nesse cenário, além de consequências econômicas, os princípios de equidade, integralidade e universalidade do SUS são infringidos e a saúde é reduzida ao acesso a insumos e serviços. ${ }^{8}$ A luz do exposto, esse artigo objetiva realizar uma revisão narrativa sobre a judicialização da saúde, em que os entes demandados judicialmente são medicamentos.

\section{REVISÃO DE LITERATURA}

O presente estudo trata-se de uma revisão narrativa desenvolvida durante o mês de janeiro de 2020, utilizando três bases de dados científicas eletrônicas: PubMed, MEDLINE e LILACS. Os descritores do Descritores em Ciências da Saúde (DeCS) e termos no Medical Subject Headings (MeSH) utilizados foram: legislation and jurisprudence AND drugs essential; judicial role AND drugs essential; patient rights AND drugs essential e jurisprudence AND drugs essential.

Para esta revisão foram incluídos os estudos publicados como artigos disponíveis na íntegra com acesso aberto, no idioma português, inglês ou espanhol e foram considerados os artigos publicados nos últimos 10 anos, incluindo 2019, ou seja, de 2010 a 2019, e que abordassem a judicialização do acesso a medicamentos. Os artigos excluídos foram aqueles não disponíveis aos pesquisadores, artigos de revisão e/ou que abordassem outros itens além dos medicamentos e duplicatas.

A partir disso, retornaram à busca 354 referências. Foi realizada a leitura do título e resumo os quais resultaram em 113 artigos incluídos e após a aplicação dos critérios de exclusão foram obtidos 91 artigos. Em seguida, procedeu-se à remoção de duplicatas que resultou em 27 publicações. Dessa forma, 27 publicações foram lidas integralmente para análise e o processo de seleção dos manuscritos está representado pelo fluxograma do estudo na Figura 1.

Para apresentação e discussão do conteúdo dos artigos foram consideradas as seguintes variáveis: autor (es), periódico e ano de publicação, local de estudo e objetivo, como mostra o Quadro 1.

Os artigos selecionados objetivam analisar o perfil do requerente e do medicamento demandado, o perfil das ações movidas contra entes estatais, a efetividade do direito à saúde, a relação entre profissionais e indústria farmacêutica, a análise das decisões judiciais, o impacto financeiro, motivos para recorrer ao poder judiciário e a reforma no processo de tomada de decisão.

Figura 1. Fluxograma dos métodos empregados para seleção de publicações na literatura.

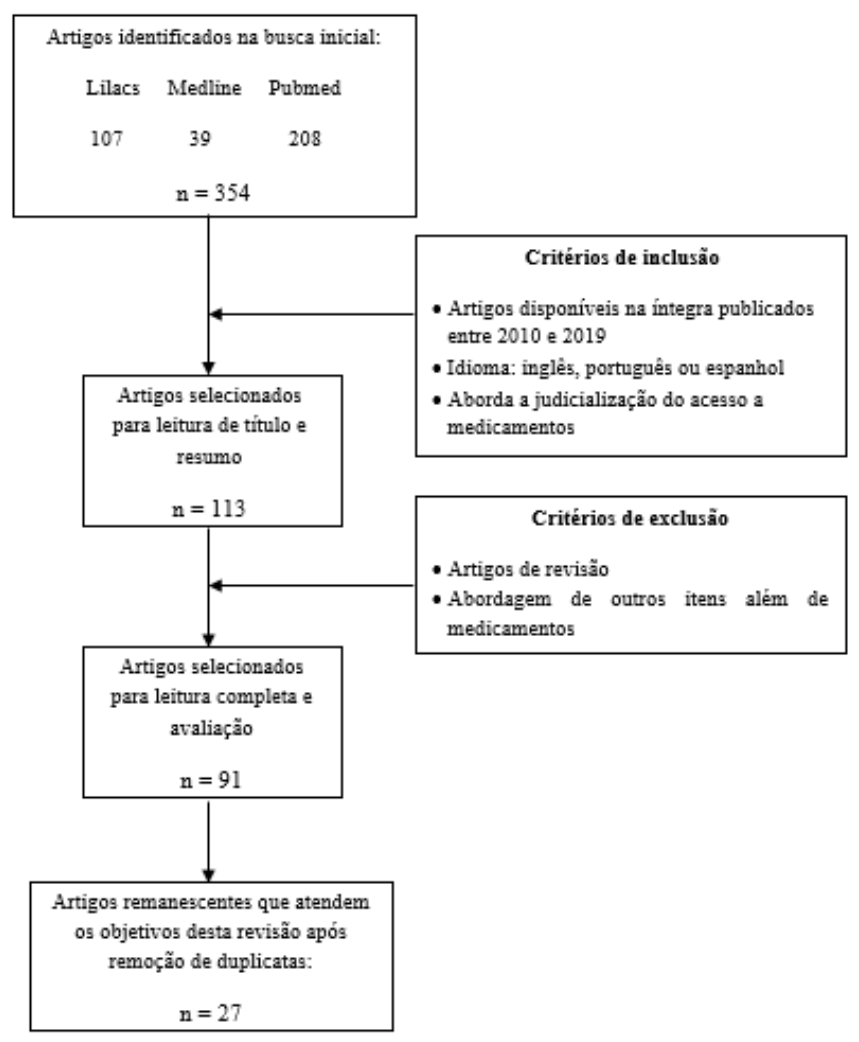


Quadro 1. Características dos estudos selecionados para leitura.

\begin{tabular}{|c|c|c|c|}
\hline Autores & Periódico/Ano & Local de estudo & Objetivo \\
\hline Campos Neto et al. ${ }^{3}$ & Rev. Saúde Pública, 2012 & Estado de Minas Gerais & $\begin{array}{l}\text { Descrever a relação entre médicos prescritores, } \\
\text { advogados e indústria farmacêutica em processos } \\
\text { para acesso a medicamentos contra o Estado. }\end{array}$ \\
\hline Lisboa, Souza $^{4}$ & Ciên. Saúde Colet., 2017 & Estado da Bahia & $\begin{array}{l}\text { Analisar as razões que levam as pessoas a } \\
\text { recorrerem ao judiciário para obter acesso às } \\
\text { insulinas análogas na Bahia. }\end{array}$ \\
\hline Biehl, Socal, Amon ${ }^{5}$ & Health Hum. Rights, 2016 & Rio Grande do Sul & $\begin{array}{l}\text { Apresentar evidência empírica que desafia e refuta a } \\
\text { ideia de anti-judicialização. }\end{array}$ \\
\hline $\begin{array}{l}\text { Chieffi, Barradas, } \\
\text { Golbaum }^{6}\end{array}$ & $\begin{array}{l}\text { BMC Health Serv. Res., } \\
2017\end{array}$ & Estado de São Paulo & $\begin{array}{l}\text { Caracterizar os processos judiciais de medicamentos } \\
\text { registrados entre } 2010 \text { e } 2014 \text { contra a Secretaria de } \\
\text { Estado da Saúde de São Paulo. }\end{array}$ \\
\hline Machado et al. ${ }^{8}$ & Rev. Saúde Pública, 2011 & Estado de Minas Gerais & $\begin{array}{l}\text { Observar o perfil dos requerentes e medicamentos } \\
\text { demandados em processos. }\end{array}$ \\
\hline $\begin{array}{l}\text { Silva, Almeida, } \\
\text { Pessôa }^{9}\end{array}$ & $\begin{array}{l}\text { Cad. Ibero-Amer. Dir. } \\
\text { Sanit., } 2017\end{array}$ & Distrito Federal & $\begin{array}{l}\text { Analisar o gasto com medicamentos judicializados } \\
\text { no âmbito do SUS no Distrito Federal. }\end{array}$ \\
\hline Pinheiro et al. ${ }^{10}$ & J. Bras. Econ. Saúde, 2019 & Belém, Pará & $\begin{array}{l}\text { Analisar gastos com insulinas análogas adquiridas } \\
\text { por via judicial no município de Belém-Pará no ano } \\
\text { de } 2016 \text {. }\end{array}$ \\
\hline $\begin{array}{l}\text { Botelho, Pessoa, } \\
\text { Lima }^{11}\end{array}$ & J. Health Biol. Sci., 2017 & Estado do Ceará & $\begin{array}{l}\text { Descrever perfil dos processos administrativos e } \\
\text { judiciais de solicitação de medicamentos à Secretaria } \\
\text { de Saúde do Estado do Ceará. }\end{array}$ \\
\hline Damasceno, Ribeiro $^{12}$ & $\begin{array}{l}\text { Cad. Ibero-Amer. Dir. } \\
\text { Sanit., } 2019\end{array}$ & $\begin{array}{l}\text { Municípios da região } \\
\text { metropolitana de Belém, } \\
\text { Pará }\end{array}$ & $\begin{array}{l}\text { Definir o cenário municipal dos processos judiciais } \\
\text { solicitados de medicamentos na região metropolitana } \\
\text { de Belém-PA. }\end{array}$ \\
\hline Trevisan et al. ${ }^{13}$ & Ciên. Saúde Colet., 2015 & $\begin{array}{l}\text { Estado do Rio Grande } \\
\text { do Sul }\end{array}$ & $\begin{array}{l}\text { Caracterizar o uso de recursos legais para obter } \\
\text { tratamento de fenilcetonúria no Rio Grande do Sul. }\end{array}$ \\
\hline $\begin{array}{l}\text { Sartori D Junior et } \\
\text { al. }{ }^{14}\end{array}$ & Ciên. Saúde Colet., 2012 & $\begin{array}{l}\text { Estado do Rio Grande } \\
\text { do Sul }\end{array}$ & $\begin{array}{l}\text { Avaliar ações judiciais que objetivam tratamento da } \\
\text { doença de Fabry no Rio Grande do Sul em } 2007 .\end{array}$ \\
\hline $\begin{array}{l}\text { Stamford, } \\
\text { Cavalcanti }^{15}\end{array}$ & Rev. Saúde Pública, 2012 & Estado do Pernambuco & $\begin{array}{l}\text { Analisar decisões judiciais no acesso a } \\
\text { medicamentos quanto a critérios de decisão, } \\
\text { pressões políticas e econômicas. }\end{array}$ \\
\hline Nisihara et al. ${ }^{16}$ & Einstein (São Paulo), 2017 & Estado do Paraná & $\begin{array}{l}\text { Descrever o perfil das ações judiciais que solicitam } \\
\text { medicamentos ajuizados na Justiça Federal do } \\
\text { Paraná. }\end{array}$ \\
\hline Biehl et al..$^{17}$ & Health Hum. Rights, 2012 & $\begin{array}{l}\text { Estado do Rio Grande } \\
\text { do Sul }\end{array}$ & $\begin{array}{l}\text { Identificar tendências em processos judiciais de } \\
\text { medicamentos no estado do Rio Grande do Sul e } \\
\text { caracterizar os demandantes. }\end{array}$ \\
\hline $\begin{array}{l}\text { Chagas, Provin, } \\
\text { Amaral }^{18}\end{array}$ & BMC Public Health, 2019 & $\begin{array}{l}\text { Capital de estado do } \\
\text { centro-oeste }\end{array}$ & $\begin{array}{l}\text { Analisar se os processos de acesso a medicamentos } \\
\text { são concernentes às políticas de saúde do Estado. }\end{array}$ \\
\hline Pepe et al. ${ }^{19}$ & Cad. Saúde Pública, 2010 & Estado do Rio de Janeiro & $\begin{array}{l}\text { Analisar ações individuais de fornecimento de } \\
\text { medicamentos no estado do Rio de Janeiro em } 2006 .\end{array}$ \\
\hline Sant'Ana et al. ${ }^{20}$ & Rev. Saúde Pública, 2011 & Estado do Rio de Janeiro & $\begin{array}{l}\text { Caracterizar os principais elementos processuais, } \\
\text { médico-científicos e sanitários que respaldam as } \\
\text { decisões judiciais de acesso a medicamentos. }\end{array}$ \\
\hline Coelho et al. ${ }^{21}$ & Rev. Saúde Pública, 2014 & Estado de Minas Gerais & $\begin{array}{l}\text { Investigar fatores relacionados à concessão de } \\
\text { liminar por medicamentos. }\end{array}$ \\
\hline Borges, Ugá22 & Cad. Saúde Pública, 2010 & Estado do Rio de Janeiro & $\begin{array}{l}\text { Discutir a atuação e o comportamento do Poder } \\
\text { Judiciário no julgamento dos processos judiciais de } \\
\text { acesso a medicamentos. }\end{array}$ \\
\hline
\end{tabular}

Continua. 
Conclusão.

Quadro 1. Características dos estudos selecionados para leitura.

\begin{tabular}{|l|l|l|l|}
\hline Autores & Periódico/Ano & Local de estudo & Objetivo \\
\hline Szpak et al. ${ }^{23}$ & Einstein (São Paulo), 2020 & Estado do Paraná & $\begin{array}{l}\text { Analisar as demandas judiciais do brometo de } \\
\text { tiotrópio para tratar doença pulmonar obstrutiva } \\
\text { crônica. }\end{array}$ \\
\hline Vidal et al. ${ }^{24}$ & Ciên. Saúde Colet., 2017 & Brasil & $\begin{array}{l}\text { Analisar processos envolvendo fornecimento } \\
\text { de drogas antineoplásicas contra entidades } \\
\text { governamentais. }\end{array}$ \\
\hline Zago et al. ${ }^{25}$ & Acta bioeth, 2016 & $\begin{array}{l}\text { Municípios do meio- } \\
\text { oeste de Santa Catarina }\end{array}$ & $\begin{array}{l}\text { Estudar ações judiciais impetradas em municípios } \\
\text { do meio-oeste de Santa Catarina para obtenção de } \\
\text { medicamentos. }\end{array}$ \\
\hline $\begin{array}{l}\text { Figueiredo, Osorio- } \\
\text { de-Castro, Pepe }{ }^{26}\end{array}$ & Cad. Saúde Pública, 2013 & Rio de Janeiro & $\begin{array}{l}\text { Analisar a demanda por medicamentos através de } \\
\text { processos judiciais na cidade do Rio de Janeiro. }\end{array}$ \\
\hline $\begin{array}{l}\text { Macedo, Lopes, } \\
\text { Barberato-Filho }{ }^{27}\end{array}$ & Rev. Saúde Pública, 2011 & Estado de São Paulo & $\begin{array}{l}\text { Analisar os pedidos judiciais para medicamentos que } \\
\text { são incorporados na assistência farmacêutica (AF). }\end{array}$ \\
\hline $\begin{array}{l}\text { Diniz, Medeiros, } \\
\text { Schwartz }\end{array}$ & Cad. Saúde Pública, 2012 & Brasil & $\begin{array}{l}\text { Analisar a dimensão financeira da judicialização } \\
\text { de três medicamentos para tratamento da } \\
\text { mucopolissacaridose. }\end{array}$ \\
\hline $\begin{array}{l}\text { Pizzzarossa, } \\
\text { Perehudoff, Forte }\end{array}$ & Health Hum. Rights, 2018 & Uruguai & $\begin{array}{l}\text { Analisar a interpretação do judiciário sobre o dever } \\
\text { do Estado no direito à saúde. }\end{array}$ \\
\hline $\begin{array}{l}\text { Loaiza et al. }{ }^{30} \\
\text { do }\end{array}$ & Health Hum. Rights, 2018 & Costa Rica & $\begin{array}{l}\text { Avaliar o processo na reforma da tomada de decisão } \\
\text { do acesso a medicamentos via judicial. }\end{array}$ \\
\hline
\end{tabular}

Para isso, os estudos recorreram à análise de processos judiciais em fóruns municipais, advocacias do Estado, cortes federais e arquivos do Ministério da Saúde. Além disso, foram realizadas consultas em bases de dados eletrônicas e análises de relatórios de consumo.

Exceto dois artigos ${ }^{9,10}$ que analisaram relatórios de consumo, processos administrativos e notas, os demais, como apresentado no Quadro 1, realizaram a análise de processos judiciais para obtenção de dados referentes à judicialização no acesso a medicamentos.

Nos estudos analisados, verificou-se aumento da incidência de processos judiciais. ${ }^{6,11}$ Em cinco artigos, os autores apresentam argumentos e motivos para a judicialização a partir de dados encontrados nos processos. ${ }^{4,12-15}$

O perfil do demandante é descrito em cinco publicações, $5,12,16-18$ sendo que ocorre tanta predominância da representação privada $^{3,6}$ quanto da representação pública. ${ }^{5,12,17,19}$ Já quanto à origem da instituição de saúde, verifica-se maior número de instituições privadas. ${ }^{3,6,8}$ Em um estudo, a relação pública e privada foi equivalente. ${ }^{20}$

Com relação ao andamento processual, verifica-se que 5 estudos $^{13,19-22}$ analisaram a concessão de liminar e antecipação de tutela, sendo constatado em apenas $u^{20}$ a exigência de comprovação da condição clínica. O tempo entre as tramitações no Poder Judiciário foram analisados por dois autores. ${ }^{13,21}$

No que tange aos medicamentos, o número de medicamentos demandados foram descritos em 14 publicações. , $^{3,-12,16,19,21,23-27}$
Já a somatória dos custos também foram descritas. .,10,14,15,23,24,25,28 Dados referentes a incorporação dos medicamentos em listas

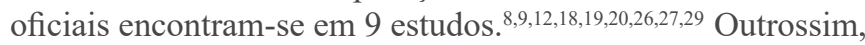
o monopólio na aquisição de medicamentos foi abordado em dois estudos. ${ }^{15,28}$ No que se refere ao fenômeno da judicialização da saúde não ser considerado um evento exclusivo da elite, há opiniões diferentes, pois há estudo ${ }^{5}$ que refere a maior litigância às classes sociais menos favorecidas, ao passo em que outro ${ }^{28}$ alega neutralidade no que tange a classes sociais.

Ademais, quanto aos recursos médicos e jurídicos utilizados na solicitação de medicamentos também há dicotomia, havendo os que recorrem apenas a recursos públicos ${ }^{8,26,30}$ quanto aqueles que utilizam de recursos privados. , $^{3,6,8}$

\section{DISCUSSÃO}

Levando em consideração os resultados encontrados e o objetivo proposto neste estudo de realizar uma revisão narrativa sobre a judicialização da saúde, em que os entes demandados judicialmente são medicamentos, observa-se maior número de publicações nos anos de 2012 e 2017 . Entretanto, nenhum fato aparente no contexto da saúde relaciona-se a este dado, sugerindo mais estudos do fenômeno da judicialização para acesso a medicamentos nestes dois anos. Destaca-se que, no entanto, os artigos foram elaborados em período anterior à data de publicação. A análise de processos datados de $1999^{3}$ evidenciam que tal fenômeno não se trata de assunto recente e vem sendo estudado não somente por pesquisadores na área da saúde, mas também por profissionais do direito, ciências econômicas e sociais. ${ }^{4,5,15,17}$ 
O principal método empregado nos estudos encontrados foi a análise de processos judiciais, tendo em vista este ser um documento que guarda todas as informações para a obtenção de medicamentos via judicial. Os dados sobre os demandantes, o objeto requerido, as decisões de autoridades judiciais e as contestações dos réus foram utilizados para a elaboração das pesquisas. O número desses documentos teve crescimento explosivo em estudo realizado entre 2002 e $2009,{ }^{17}$ demonstrando a expansão e preocupação com o fenômeno.

Três estudos mostram que o perfil dos demandantes de medicamentos são pessoas provenientes de classes sociais menos favorecidas e sem condições de adquirir os medicamentos prescritos. ${ }^{5,17,18}$ Dessa forma, a judicialização é entendida como uma maneira de garantir o direito à saúde e, para alguns indivíduos, a última medida para obter acesso a serviços de saúde. ${ }^{8}$

Concomitante a isso, há opiniões paradoxais no que se refere à origem pública ou privada da representação jurídica e médica e sua relação com a classe social do requerente. Isto é, estudos relatam pacientes com condições financeiras de arcar com custos referentes à representação jurídica e serviços de saúde. ${ }^{3,6,8}$ Ao passo que, há também aqueles que utilizam apenas recursos públicos, ${ }^{8,26,30}$ inferindo que o fenômeno não é homogêneo, ou seja, tanto há pessoas que realmente necessitam dos serviços públicos, quanto pessoas com condições de arcar com essas despesas. Ademais, a presença de representação pública e prescrição por médicos particulares refletiria a morosidade do sistema de saúde e urgência do tratamento, o que leva muitos pacientes recorrerem a serviços médicos privados apenas para adquirir o receituário necessário para a litigância.

Nesse contexto, no que concerne ao conhecimento de direitos cidadãos sobre acessibilidade aos serviços e insumos de saúde deve-se considerar o princípio de equidade, designado pela adaptação visando critérios de justiça, ou seja, acolhendo todos os cidadãos de acordo com suas necessidades biopsicossociais. $\mathrm{O}$ princípio da equidade não é visualizado no que tange o acesso ao conhecimento, pois classes sociais menos abastadas têm menor acesso à informação acentuando disparidades na validade da universalidade à saúde e à justiça. ${ }^{3}$

Entre os medicamentos judicializados, verifica-se que estão presentes os que já pertencem a listas oficiais de distribuição da Assistência Farmacêutica. Fato este relacionado à ineficiência na distribuição atribuída a problemas de disponibilidade e logística, levando o paciente a buscar o Poder Judiciário para obter o medicamento. ${ }^{3}$ A presença ou ausência e incorporação dos medicamentos em listas oficiais governamentais foi abordada em 8 estudos, ${ }^{8,9,18,19,20,23,26,27}$ e pode-se constatar que a primeira opção se revelou mais eficiente no que tange à economia e custo-benefício a longo prazo. Exemplos disso foram a incorporação da insulina glargina à relação da Secretaria de Estado de Saúde de Minas Gerais (SES/MG) em 2005, adalimumabe e etanercepte ao Programa Nacional de Melhoria do Acesso e da Qualidade da Atenção Básica (PMAC, protocolo da artrite reumatoide) em 2006 e dos fármacos bosentana e sildenafil à SES/MG em 2009, após várias solicitações judiciais destes medicamentos. ${ }^{8}$

Tal fato é destacado pelo exemplo exposto no estudo de Silva et al (2017), ${ }^{9}$ em que a incorporação da alfaglicosidase ao SUS em 2014 gerou queda nos gastos com a judicialização deste: de R\$ 2.153.732,50 em 2015 para $\mathrm{R} \$ 637.198,97$ de janeiro a agosto de 2016. Dada a importância disso, oito estudos 9,10,14,15,23,24,25,28 $^{2}$ discorreram sobre os custos de medicamentos e seu impacto financeiro, além de 12 publicações s,8, $^{3,10,16,19,21,23-27}$ dissertarem sobre a demanda de medicamentos.

Ademais, pode-se afirmar o estabelecimento de uma relação contestada entre a judicialização da saúde e a indústria farmacêutica. Há estudo ${ }^{6}$ que afirma um elevado número de ações judiciais em que o demandante recorre a um número reduzido de profissionais liberais médicos e advogados, o que pode sugerir uma correlação com a indústria de medicamentos. Entretanto, o presente estudo reconhece esta limitação do estudo e considera que é necessário um aprofundamento maior para afirmar esta correlação.

Neste raciocínio e considerando a totalidade de concessão de liminares por parte dos juízes neste estudo e a necessidade em alguns casos de comprovação clínica patológica, ${ }^{21}$ uma vez que tais não apresentam formação acadêmica médica, acabam por designar um médico perito para avaliar indicação da prescrição ou avaliam os argumentos daquele que assiste o demandante, sempre tomando por base documentos médicos e não jurídicos. ${ }^{16}$ Os magistrados têm de julgar um parecer médico sem apresentar profundo conhecimento sobre tais causas $^{16}$ o que pode influenciar no julgamento.

Em relação às finanças, observa-se uma preocupação com o impacto negativo do fenômeno nas finanças da União, Estados e municípios ${ }^{7}$ ao comprometer parte significativa do montante de verbas destinadas ao setor da saúde, pois envolvem gastos não previstos nos planos orçamentários, além de atender uma população específica em detrimento da coletividade. $\mathrm{O}$ atual funcionamento do sistema com recursos financeiros limitados associado com a necessidade de realocação de verbas gerada pela judicialização e impasses éticos, tornam o assunto polêmico e alvo de discussões aos atores envolvidos.

\section{CONSIDERAÇÕES FINAIS}

Na revisão narrativa realizada há o apontamento de discussões referentes ao impacto negativo causado pela judicialização da saúde nas finanças públicas da saúde, bem como a afirmação de que esse mecanismo se configura como um meio de acesso à saúde e seus insumos para os demandantes. Destaca-se a necessidade de atualização das listas oficiais, bem como melhor disponibilização dos fármacos já vigentes à população.

Assim, devido ao crescimento do fenômeno nas últimas décadas, de seu impacto socioeconômico cultural e da crise vivenciada pelo Brasil, torna-se imprescindível o desenvolvimento de novos estudos sobre o assunto para aprofundamento do conhecimento e proposição de estratégias para fortalecimento do direito à saúde. 


\section{REFERÊNCIAS}

1. Brasil. Presidência da República. Casa Civil. Subchefia para Assuntos Jurídicos. Constituição da República Federativa do Brasil de 1988 [Internet]. Brasília; 1988 [acesso em: 15 ago 2020]. Disponível em: http://www.planalto.gov.br/ccivil_03/Constituicao/ Constituicao.htm.

2. Ventura M, Simas L, Pepe VL, Schramm FR. Judicialização da saúde, acesso à justiça e a efetividade do direito à saúde. Physis. 2010;20(1):77-100.

3. Campos OH Neto, Acurcio FA, Machado MA, Ferré F, Barbosa FL, Cherchiglia ML, et al. Médicos, advogados e indústria farmacêutica na judicialização da saúde em Minas Gerais, Brasil. Rev Saúde Pública. 2012;46(5):784-90.

4. Lisboa ES, Souza LE. Por que as pessoas recorrem ao judiciário para obter o acesso aos medicamentos? O caso das insulinas análogas na Bahia. Cien Saude Colet. 2017;22(6):1857-64.

5. Biehl J, Socal MP, Amon JJ. The judicialization of health and the quest for state accountability: evidence from 1,262 lawsuits for access to medicines in southern Brazil. Health Hum Rights. 2016;18(1):209-20.

6. Chieffi AL, Barradas RC, Golbaum M. Legal access to medications: a threat to Brazil's public health system? BMC Health Serv Res. 2017;17(1):499.

7. Reis AA, Sóter AP, Furtado LA, Pereira SS. Tudo a temer: financiamento, relação público e privado e o futuro do SUS. Saúde debate. 2016;40(spe):122-35.

8. Machado MA, Acurcio FA, Brandão CM, Faleiros DR, Guerra AA Júnior, Cherchiglia ML, et al. Judicialização do acesso a medicamentos no Estado de Minas Gerais, Brasil. Rev Saúde Pública. 2011;45(3):590-8.

9. Silva EM, Almeida KC, Pessoa GS. Análise do gasto com judicialização de medicamentos no Distrito Federal, Brasil. Cad Ibero-Amer Dir Sanit. 2017;6(1):112-26.

10. Pinheiro PN, Garcia JV, Cardoso ET, Luz DA, Silva VG. Farmacoeconomia: gastos com análogos de insulina adquiridos por meio de judicialização em um município do estado do Pará, Brasil, no ano de 2016. J Bras Econ Saúde. 2019;11(1):42-8.

11. Botelho PA, Pessoa NT, Lima AM. Direito à saúde: medicamentos mais solicitados por demandas administrativas e judiciais por uma secretaria estadual de saúde no biênio de 2013 a 2014. J Health Biol Sci. 2017;5(3):253-8.

12. Damasceno TV, Ribeiro KD. Judicialização da saúde nos municípios da região metropolitana de Belém-PA. Cad Ibero-Amer Dir Sanit. 2019;8(2):1-132.

13. Trevisan LM, Nalin T, Tonon T, Veiga LM, Vargas P, Krug BC, et al. Access to treatment for phenylketonuria by judicial means in Rio Grande do Sul, Brazil. Ciên Saúde Colet. 2015;20(5):160716 .

14. Sartori D Junior, Leivas PG, Souza MV, Krug BC, Balbinotto G, Schwartz IV. Judicialização do acesso ao tratamento de doenças genéticas raras: a doença de Fabry no Rio Grande do Sul. Ciên Saúde Colet. 2012;17(10):2717-28.
15. Stamford A, Cavalcanti M. Legal decisions on access to medicines in Pernambuco, Northeastern Brazil. Rev Saúde Pública. 2012;46(5):791-99.

16. Nisihara RM, Possebom AC, Borges LM, Shwetz AC, Bettes FF. Judicial demand of medications through the Federal Justice of the State of Paraná. Einstein (São Paulo). 2017;15(1):85-91.

17. Biehl J, Amon JJ, Socal MP, Petryna A. Between the court and the clinic: lawsuits for medicines and the right to health in Brazil. Health Hum Rights. 2012;14(1):36-52.

18. Chagas VO, Provin MP, Amaral RG. Administrative cases: an effective alternative to lawsuits in assuring access to medicines? BMC public health. 2019;19(212):1-10.

19. Pepe VL, Ventura M, Sant'ana JM, Figueiredo TA, Souza VR, Simas L, et al. Caracterização de demandas judiciais de fornecimento de medicamentos "essenciais" no Estado do Rio de Janeiro, Brasil. Cad Saúde Pública. 2010;26(3):461-71.

20. Sant'Ana JM, Pepe VL, Figueiredo TA, Osorio-de-Castro CG, Ventura M. Racionalidade terapêutica: elementos médico-sanitários nas demandas judiciais de medicamentos. Rev Saúde Pública. 2011;45(4):714-21.

21. Coelho TL, Ferré F, Campos OH Neto, Acurcio FA, Cherchiglia ML, Andrade EI. Legal and health variations in drug litigation injunctions granted in Minas Gerais. Rev Saúde Pública. 2014;48(5):808-16.

22. Borges DC, Ugá MA. Conflitos e impasses da judicialização na obtenção de medicamentos: as decisões de $1^{\text {a }}$ instância nas ações individuais contra o Estado do Rio de Janeiro, Brasil, em 2005. Cad Saúde Pública. 2010;26(1):59-69.

23. Szpak R, Strapasson GC, Böger B, Rattmann YD, Gomes EC. Legal demands of the tiotropium bromide for treatment of chronic obstructive pulmonary disease and their financial impact for the State of Paraná, Brazil. Einstein (São Paulo). 2020;18:17.

24. Vidal TJ, Moraes EL, Retto MP, Silva MJ. Demandas judiciais por medicamentos antineoplásicos: a ponta de um iceberg? Ciên Saúde Colet. 2017;22(8):2539-48.

25. Zago B, Swiech LM, Bonamigo EL, Schlemper BR Junior. Aspectos bioéticos da judicialização da saúde por medicamentos em 13 municípios no meio-oeste de Santa Catarina, Brasil. Acta Bioeth. 2016;22(2):293-302.

26. Figueiredo TA, Osorio-de-Castro CG, Pepe VL. Evidencebased process for decision-making in the analysis of legal demands for medicines in Brazil. Cad Saúde Pública. 2013;29(supl.1):15966.

27. Macedo EI, Lopes LC, Barberato-Filho S. Análise técnica para a tomada de decisão do fornecimento de medicamentos pela via judicial. Rev Saúde Pública. 2011;45(4):706-13.

28. Diniz D, Medeiros M, Schwartz IV. Consequências da judicialização das políticas de saúde: custos de medicamentos para as mucopolissacaridoses. Cad Saúde Pública. 2012;28(3):47989. 
29. Pizzarossa LB, Perehudoff K, Forte JC. How the Uruguayan judiciary shapes access to high-priced medicines: a critique through the right to health lens. Health Hum Rights. 2018;20(1):93-105.
30. Loaiza OR, Morales S, Norheim OF, Wilson BM. Revisiting health rights litigation and access to medications in Costa Rica: preliminary evidence from the Cochrane Collaboration Reform. Health Hum Rights. 2018;20(1):79-91.

\section{Como citar:}

Hawerroth MG, Sonoda LY, Andrade RD, Santos JS, Maia MA. O acesso à medicamentos: aspectos da judicialização da saúde. Rev Med UFC. 2021;61(1):1-7. 